\title{
Research on Control Technology of Concentrated Coal Mine Rail Transport Intelligence
}

\author{
Guodong Song ${ }^{1,2,3}$, Xiaohui Yang ${ }^{1,2,3}$, Jianfeng Gao ${ }^{1,2,3}$, Wenzhen Wu ${ }^{1,2,3}$ \\ 1.China Coal Research Institute, BeiJing 100013,China; \\ 2.State Key Laboratory of Coal Resource Efficient Mining and Clean Utilization, BeiJing 100013,China; \\ 3.Beijing Mine Safety Engineering Technology Research Center, BeiJing 100013,China
}

\begin{abstract}
Based on a matrix of interlocking control strategy, industrial plc control technology, field bus communication technology of mine shaft orbit transportation intelligent centralized control technology and implementation method are introduced in this paper. Line operation using the multi section partition method to draw intuitive control strategy, simplifying the interlock logic, to realize the intelligent management and dispatching of the railway train, improve transport efficiency; application of plc and fieldbus technology improves the stability and reliability of the system operation, convenient underground site operation personnel and ground remote scheduling scheduling staff to keep abreast of the train running state, issued instruction scheduling, to ensure the smooth of mine transportation operation. Promote the automation and intelligent development of coal transportation, to improve the level of information technology in coal mine production scheduling, decision-making is of great significance.
\end{abstract}

\author{
KEYWORDS \\ Route interlocking \\ $\mathrm{Plc}$
}

Field bus

Mine transportation

\section{INTRODUCTION}

Coal mine safety production is the problem the party and the government attaches great importance, it not only relates to the healthy development of coal industry, is also the premise and guarantee for building a harmonious society. According to 2011 statistics, the safety accident casualties in rail transport of coal mine accounted for $20 \%$ of the total casualties of coal production accidents, is second to the roof accident disaster.

The coal mine railway intelligent transportation control system has low vehicle identification precision, complex transportation vehicle scheduling and other features. Through studying the coal mine rail transport vehicle intelligent recognition technology, vehicle video linkage and early warning technology, control technology of intelligent transportation set, configuration software monitoring technology, a set of safe, reliable coal mine rail transport control device, with monitoring location, transportation scheduling, alarm linkage, and other functions, as well as terminal display and analysis capabilities such as voice, image, data was developed. It can achieve crash warning, underground vehicle's optimum scheduling, achieve automatic and intelligent underground transportation.

Copyright (C) 2016 Guodong Song, Xiaohui Yang, Jianfeng Gao and Wenzhen Wu doi: $10.18686 /$ esta.v3i1.7

This is an open-access article distributed under the terms of the Creative Commons Attribution Unported License (http://creativecommons.org/licenses/by-nc/4.0/), which permits unrestricted use, distribution, and reproduction in any medium, provided the original work is properly cited.

\section{Characteristics of control object}

Coal mine underground rail transport generally includes three categories of the gate rail, inclined lane track and cross track. First, for entry rail transportation, the main monitoring objects include signal equipment, switch machine, locomotives and other equipment, running state monitoring object information real-time feedback to the locomotive electronic control device, a control instruction is sent, to ensure its safe operation in orbit drift; second, for entry rail transportation, main monitoring objects include signal, hoist, wire rope, sports protective gear and other equipment, running state monitoring object information real-time feedback to the locomotive electronic control device, a control instruction is sent, ensure the safe operation in inclined lane orbit; finally, for the cross track, main monitoring objects are switch machine and the bidirectional locomotive, the locomotive running state information are feedback to each other locomotive electronic control device, a control instruction is sent, in order to avoid locomotive collision accident. 
Coal mine slope rail transport intelligent control technology to comprehensively consider the running characteristics of the above three kinds of orbit and accused of equipment, with wireless network field bus control structure industrial control network as the core of locomotive transportation safety monitoring and automatic dispatching system, it relies on wireless access network testing vehicle running status, controlled by the nearby races to transportation dispatching center, displayed on the graphic display device to simulate a figure, for scheduling staff to grasp, and rely on the computer a powerful memory, judgment and operation ability, will dispatch personnel scheduling intent is decomposed into specific control instruction, control equipment switch position and achieve route open such as scheduling, in order to ensure transportation safety, improve command efficiency, and increase the economic benefit.

\section{Control strategy}

During railway transportation, first shunting demand, based on the actual traffic situation will be divided into several sections, transportation lines each section at the same time allow only one train running or take up, in front of the train to leave after this section, the rear train is allowed to enter, so as to ensure the safety of the train operation, section approach is the most basic rail transport monitoring system of the control unit. Section division and access control is shown in figure 1 .

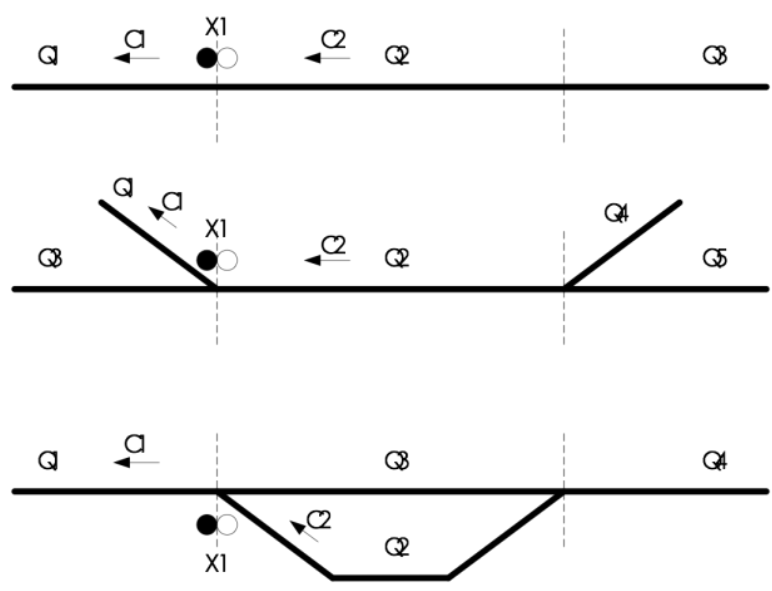

Figure 1 section division and access control

Wherein, q1-5 represents sections, the $x 1$ represents the signal number, $c 1-2$ represents the train number. Diagram, q1 section $\mathrm{c} 1$ train is running or occupy, signal is prohibited to other trains into q1 section; train operation starting point to the end of the line is composed of several end to end section, under the condition of the vehicles require high dense, scheduling, can be appropriate to minimize the length of each section, in order to improve the efficiency of train transportation.

In the process of train dispatching, it is needed to define each train route, to scheduling application, obtaining the route application signal; in the approach, the mutual restriction relation between signal, rail is the premise of train safety, the restrict relationship is called "interlocking", is an expression of transportation decomposition, the route path, application approach, hostile approach, signal open state, switch position comprehensive analysis, which is the train dispatching personnel optimization scheduling need to master a dynamic relational table, and the basis for forming the computer monitoring and programming.

Considering 4 access as example, route j1 occupied sections in turn for q2, q4, approach j2 occupied sections in turn in q3, q2 and q1, approach j3 occupied sections in turn q5, q7, q9, q11, route j4 ordinal take up section $q 11, q 10$, each access is expressed as the form of a matrix :

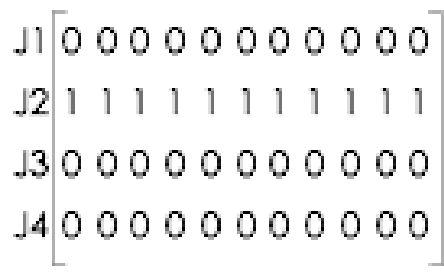

To the dot product gain matrix and its transposed matrix operation, get the matrix :

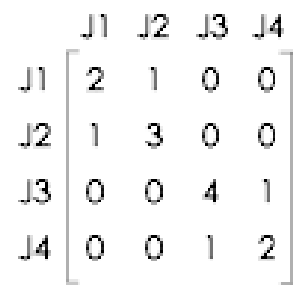

Matrix of the diagonal line on the value of each route to take up the number of extents, not on the diagonal non-zero value corresponding to each route has a hostile relationship and jointly take up on the number of extents, 0 means two route without sharing section. The access condition can be the basis of control program compiled according to logical relationship.

\section{3 .System design}

With the development of plc technology in the field of industrial control and application, its increasingly prominent feature of high integration, good stability, and combining with rail transport lines long, high real-time demand and complex control logic, this paper uses the plc as control core, using siemens industrial communication network in two levels of industrial ethernet and profibus-dp fieldbus control network topology. The upper adopts industrial ethernet, for communication between controller and ground equipment. Lower the profibus-dp fieldbus, used in the controller and the position sensor signal, switch machine, such as communication between field devices, system topology structure as shown in figure 2. 


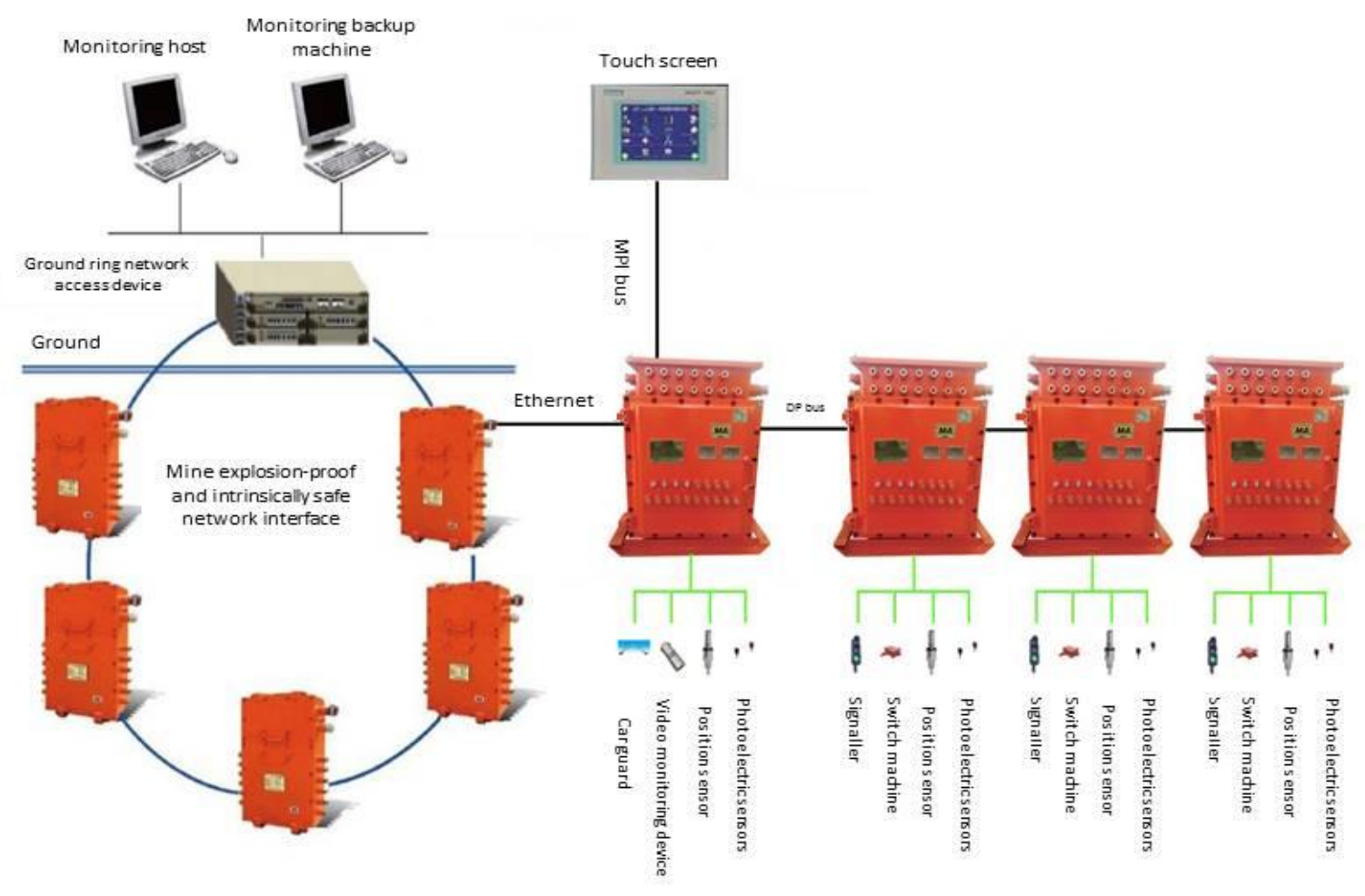

Figure 2 topology of rail transport set control system

The core of system design is the the software implementation of control strategy, must strictly meet the requirements of the train scheduling, on-site operator approach when the signal is given, in order to detect enemy into the road, the front section takes up state and position switch, judge whether meet the requirements of process route. After being into the road conditions, open up and signal, release for train travel, at the same time in the train constantly in the process of inspection on the above conditions, in order to determine whether it meets the road condition and the signal open condition.

Except for the above basic process logic, control program according to the priority scheduling and process requirements set into the road, principles of mainly includes three aspects, one is inclined lane transportation priority to lane, the steep inclined lane transport, in the event of sports car serious consequences, so you need to give inclined lane transportation higher priority rights; secondly, on the scheduling priority remote scheduling, downhole environment complex, uncertainties more, the scene can better grasp the current actual working condition, so should be paid to the scheduling of higher priority privileges; thirdly, consequent route takes precedence over the reverse route, can avoid frequent stops and starts running trains, to improve the overall efficiency of transportation.

\section{Conclusion}

Wang po coal mining in shanxi, including inclined lane transportation rail used into jobs lock control strategy, realize intelligent centralized monitoring of the underground rail transport, proved that the control strategy of rigor, satisfy the operating personnel in mine and to meet the requirements of the ground dispatch personnel to train transportation scheduling, and enhance the automation of the railway transportation.

Funds: special national international technology cooperation (2014dfr70500) 


\section{References}

1. Gao Jianping. Plc application in the mine letter sets closed system [j]. Journal of nonferrous metallurgical design and research. 2000, (6) : 9-14.

2. Pang Xun, li wei, yan leipeng, etc. Letter sets closed system into jobs lock table automatic generation algorithm research [j]. Journal of mining research and development. 2008, 28 (3) : 40-42.

3. Huagang, Zuo Ming. Rail transport monitoring system modeling and control algorithm of research [j]. Journal of china university of mining. 1998, 27 (1) : 90-94.

4. Jia ShiSheng. The research and design of monitoring and control system for mine railway transportation [d]. Shandong university of science and technology. 2007.

5. Lan Xianchen, Zuo Ming, Hua Gang. [m]. Monitoring and control system for mine railway transportation of china university of mining press, 1992. 11-30.

6. Zheng Changxiang. Based on profibus-dp mine rail transport "letters" closed "monitoring system design [j]. Journal of coal mine machinery. 2012, 33 (4) : 234-235.
7. Wang Baode. The coal mine transport intelligent dispatching command system [j]. Industrial automation. 2014, 40 (7) : 87-89.

8. Liu Zhenyang, Ji Zengquan. Kj221 type locomotive transport in coal mine monitoring and control system [j]. The application of coal mine safety. 2014, 45(2) : 97-99.

9. Lu Weifeng. Track letter sets of coal mine based on the dcs, closed system [j]. Coal mine mechanical and electrical. 2015 (1) : 78-81.

10. Li Junhong. Mine electric locomotive security system design [j]. Industrial automation. 2016, 42 (6) : 77-79.

\section{Author introduction}

Song guodong(1984 -), xuzhou, jiangsu province, assistant researcher, master degree, graduated from northeastern university in 2009, the current coal science and technology research institute co., ltd. Department of automation equipment branch, mainly engaged in the scientific research work of coal mine integrated automation and so on, published 9 papers 